\title{
Metabolic and Growth Inhibition of Mycoplasma gallisepticum by Antiserum
}

\author{
By G. N. WOODE* AND D. A. MCMARTIN \\ Department of Veterinary Pathology, Royal (Dick) School of Veterinary Studies, \\ Summerhall Square, Edinburgh and \\ Ministry of Agriculture, Fisheries and Food, Veterinary Laboratory, \\ Eskgrove, Lasswade, Midlothian
}

(Received 7 December 1970; revised 7 August 1972)

\begin{abstract}
SUMMAR Y
Chicken antisera to the s6 strain of Mycoplasma gallisepticum were used to study the mechanism of metabolic inhibition and growth inhibition by specific antibody. In high dilutions of antiserum the lag phase of growth was significantly prolonged, but eventually growth commenced and proceeded normally. In low dilutions of antiserum, growth was permanently inhibited. The prolongation of the lag phase was greater in a medium containing $10 \%$ horse serum than in $10 \%$ swine serum; with chicken serum in the medium the lag phase was of normal duration. The results correlated with the observation that the metabolic inhibition (m.i.) titres of antisera were higher when the test was conducted in horse serum than in swine or chicken serum. The antigens on the organism associated with the inhibition of growth by antiserum appeared to be essential physiologically active 'receptors'. Antisera to growth medium caused agglutination of the organism, but did not inhibit growth or metabolism.
\end{abstract}

\section{INTRODUCTION}

Following intranasal inoculation of Mycoplasma gallisepticum, immunity to the effect of air sac challenge was demonstrated although the mechanism of this resistance was not determined (McMartin \& Adler, I96I). As a preliminary to investigations on this immunity, the role of antibody in inhibiting growth of the organism was investigated. This paper reports on the effect on growth of the organism by antibody, and correlates this with the metabolic inhibition (m.i.) titre. To evaluate the importance of antibody in vivo, attempts were made to distinguish the antigens associated with agglutination from those involved in inhibition of growth by antisera.

\section{METHODS}

Mycoplasma strain. The s6 strain (Zander, 196I) of Mycoplasma gallisepticum was used.

Mycoplasma media. The growth medium was based on bacto PPLO broth (Difco, without crystal violet), and bacto PPLO agar (Difco; Difco Laboratories, Detroit, Michigan, U.S.A.). In some experiments this broth was replaced by a chicken broth prepared by the method of Jordan \& Kulasegaram (1968), except that nicotinamide-adenine dinucleotide was excluded. To the broth and to the agar were added the following constituents: $0.3 \%$ glucose, $0.5 \%$ yeast hydrolysate (Albimi Laboratories, New York, U.S.A.), penicillin at 500 i.u./ml (Glaxo Laboratories, Greenford, Middlesex), thallous acetate at $0.05 \%$, and phenol red at $0.002 \%$. With the exception of yeast extract these additives were also added to

* Present address: Institute for Research on Animal Diseases, Compton, nr Newbury, Berkshire. 
the chicken broth. Finally $10 \%(\mathrm{v} / \mathrm{v})$ animal serum was added to the medium. The serum used varied according to the experiment from one of the following: horse serum (HS) (no. 3 Burroughs Wellcome \& Co. Ltd, London), swine serum (SS) and chicken serum (CS). The latter two sera were prepared in this laboratory from freshly killed animals and sterilized by membrane filtration (APD $0.2 \mu \mathrm{m}$; Sartorius, V. A. Howe Ltd, London). The chicken serum was taken from a flock of chickens known to be free from Mycoplasma gallisepticum.

Antigen production. Strain 56 was cloned three times by selecting a single colony from a culture grown on agar and growing the organism in HS broth. This culture was then grown on agar and the selection procedure repeated. Finally an HS broth culture was prepared and the organisms harvested when in the logarithmic phase of growth. The suspension was stored in $0.5 \mathrm{ml}$ volumes in vials at $-65^{\circ} \mathrm{C}$.

Antiserum production. Antisera were prepared in chickens free of Mycoplasma gallisepticum by the intranasal inoculation of $0.5 \mathrm{ml}$ of living organisms at a concentration of $\mathrm{IO}^{7}$ colour-changing units (c.c.u.)/ml (Taylor-Robinson \& Berry, I969) followed three weeks later with $\mathrm{I} \cdot 0 \mathrm{ml}$ of the same material inoculated into the left abdominal air sac. After a further two weeks the birds were bled and the sera harvested and heat inactivated at $56{ }^{\circ} \mathrm{C}$ for $30 \mathrm{~min}$. Antiserum against antigens in the medium was prepared by twice-weekly intravenous inoculations of $0.5 \mathrm{ml}$ of medium, for a period of three weeks, and serum obtained one week later. Antiserum to the inactivated organism was made as follows: the organisms were washed three times with phosphate-buffered saline, $\mathrm{pH} 7 \cdot 2$, and concentrated to twice the opacity of a no. Io Wellcome Opacity tube (Burroughs Wellcome \& Co. Ltd, London) and inactivated with merthiolate at $\mathrm{I} / 10000$ dilution. Antiserum was then prepared by twice-weekly subcutaneous inoculations of $0.5 \mathrm{ml}$ of suspension for three weeks.

Metabolic inhibition (m.i.) test. The method of Taylor-Robinson \& Berry (I 969) was employed, with the following modifications. Opaque white plastic plates (PVC), containing 96 cavities each measuring $16 \mathrm{~mm}$ in diam. and $10 \mathrm{~mm}$ in depth were used (Manuplastics Ltd, Southdown Works, London, S.W. 20). The plates were washed in Quix (Lever Industrial Ltd, London), and rinsed in six changes of de-ionized distilled water. Dried plates were exposed to ultraviolet irradiation (Hanovia Lamps, Slough) for one hour prior to use. The plates were used repeatedly after inactivation of the organism in $2.0 \%(\mathrm{v} / \mathrm{v})$ chloros.

Antisera were diluted in twofold steps in the relevant growth medium, using $0.25 \mathrm{ml}$ of diluent in each well. The antigen suspension was diluted to $10^{4}$ c.c.u., from a culture harvested in the exponential phase of growth, and $0.25 \mathrm{ml}$ vol. added to each well. The titre for this organism suspension was confirmed for each m.i. test. A standard positive serum and a standard negative serum were titrated on each occasion as controls. The m.i. titre was read when all the dilutions of the negative serum titration were showing a colour change from red to yellow, indicating growth of the organism. At this time the dilution of the antiserum which showed o to $50 \%$ colour change of the medium was taken as the end-point and called the 'early' m.i. titre. On further incubation, dilutions of the antiserum which had shown no colour change, progressively turned yellow. Finally, permanent inhibition of growth was shown by lack of a colour change in a low dilution of the antiserum and this was called the 'final' m.i. titre. Six different antisera were repeatedly tested. The early and final m.i. titres were reproducible and showed only a twofold variation around their mean titres. The antisera used had mean early m.i. titres within the range of $\mathrm{I} / 640$ to $\mathrm{I} / 2560$, and final $\mathrm{m}$.i. titres of $I / 5$ to $I / 40$ respectively. The difference between the early and final $m$.i. titre for each antiserum was consistent, in five cases being 64 -fold, and in one 128-fold. Antisera of low potency produced more variable results because of the growth-promoting effect of normal chicken serum components in antisera. All manipulations were conducted inside a hood 
which had been ultraviolet light-irradiated for one hour before use. A dilution of organisms, which in control cultures grew into a hundred colonies, showed complete inactivation after exposure to irradiation for $\mathrm{I} 20 \mathrm{~s}$.

Growth measurement. Organisms in one of the growth media under test were incubated at $37^{\circ} \mathrm{C}\left( \pm 0 \cdot \mathrm{I}^{\circ} \mathrm{C}\right)$ in a water bath. Samples were removed at intervals, diluted by tenfold steps in broth, and two drops (pipette calibrated to deliver $30 \mathrm{drops} / \mathrm{ml}$ ) of each dilution inoculated on to each of two agar plates. The plates were left at room temperature for $30 \mathrm{~min}$, and then inverted and incubated in a humidified sealed box, in air, at $37^{\circ} \mathrm{C}$. After seven days of incubation the total number of colonies was counted, and the mean count/drop determined. One colony was taken to represent one colony-forming unit (c.f.u.) in the original inoculum. This technique was used to test the effect of penicillin, thallous acetate and phenol red on growth. Over twenty-four hours no difference could be detected between cultures with and without these additives. The accuracy of the technique was determined by titrating the organism harvested when in the logarithmic growth phase. For a threefold dilution, the number of c.f.u. declined by approximately threefold, with a 10 to $15 \%$ error representing the sampling and technical errors in the method. Replicate titrations of the same culture were made, using one of the following diluents: $10 \%$ HS broth, broth only, and phosphatebuffered saline, $\mathrm{pH} 7 \cdot 2$. No significant difference was observed in the number of c.f.u. $\left(270 \times \mathrm{IO}^{3}\right.$ to $3 \mathrm{IO} \times \mathrm{IO}^{3}$; standard deviation $\left.15 \cdot 3\right)$ determined with these diluents.

Agglutination test. An agglutinating antigen was prepared from organisms grown in the media, HS broth, SS broth and CS chicken broth, following the method of Adler (I958), except that crystal violet was excluded. Organisms were washed with phosphate-buffered saline $(\mathrm{pH} \mathrm{7.2)}$ three times and concentrated to twice the opacity of a no. Io Wellcome opacity tube, in phosphate buffer with methiolate at a final dilution of I/I0000.

A rapid slide agglutination test using this antigen was carried out with various antisera on glass over a light source (Adler, I954).

\section{RESULTS}

Antigenic variation. Early inhibition of growth by high dilutions of antiserum (early m.i. titre) was followed by growth of the organism. In order to investigate whether this was due to the presence of organisms showing antigenic variation, a culture was grown in the HS broth, with addition of antiserum at a final dilution of $\mathrm{I} / \mathrm{ro00}$, and organisms harvested when a $50 \%$ colour change was observed. A comparative m.i. test with these organisms and the standard suspension against the above antiserum, gave titres of I/I 280 against both suspensions, indicating that growth of the organisms in antiserum was not due to the presence of antibody-resistant variants.

Growth of the organism in different dilutions of antiserum. In the m.i. test the growth rate of the organism in the presence of antiserum was correlated with the dilution of antiserum. To investigate this, replicate cultures of the organism, sampled in the logarithmic phase of growth, were prepared containing different dilutions of antiserum. At intervals samples were removed and titrated (Fig. I). The number of samples taken was insufficient to determine a detailed growth curve but the results showed that the time to reach a population size of $10^{6} \mathrm{c}$. f.u. $/ \mathrm{ml}$ increased with increasing antiserum concentration.

Effect of normal serum source on m.i. and growth inhibition by antiserum. The organism grew well in the presence of HS, SS, or CS in broth but when these different growth media were used for the m.i. test, the early m.i. titres of antisera varied with the serum used. The titre was highest with broth containing HS (Table I). Growth studies were then made to determine which part of the growth curve was influenced by the use of these normal sera in the 


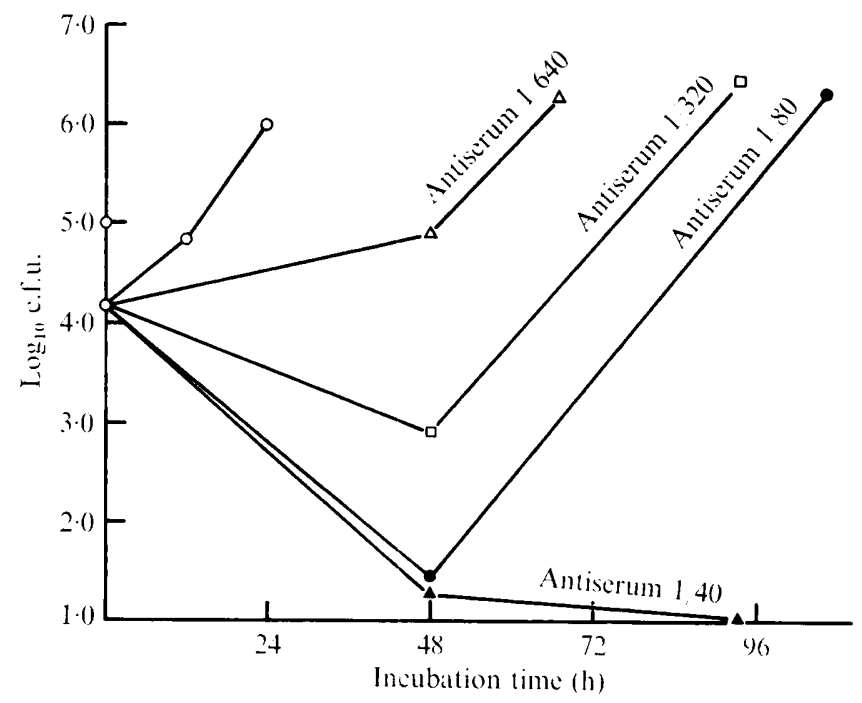

Fig. I. Growth of Mycoplasma gallisepticum in different dilutions of antiserum. $\bigcirc$, Control culture without antiserum; $\triangle$, antiserum at $\mathrm{I} / 640$ dilution; $\square$, antiserum at $\mathrm{I} / 320$ dilution; $\mathbf{O}$, antiserum at $\mathrm{I} / 80$ dilution; $\boldsymbol{\Delta}$, antiserum at $\mathrm{I} / 40$ dilution.

Table I. Influence of normal serum present in medium on m.i. titre

$\begin{array}{ccc}\text { Antiserum } & \begin{array}{c}\text { Normal } \\ \text { serum }\end{array} & \text { M.i. titre } \\ 1869 & \text { HS } & 2560 \\ & \text { SS } & 640 \\ & \text { CS } & 40 \\ & \text { HS } & 1280 \\ & \text { SS } & 320 \\ & \text { CS } & 80 \\ & \text { HS } & 1280 \\ 5371 & \text { SS } & 320 \\ & \text { CS } & 80 \\ 5390 & \text { HS } & 2560 \\ & \text { CS } & 320 \\ & \text { HS } & 2560 \\ & \text { CS } & 160\end{array}$

M.i. titre is reciprocal of the highest chicken antiserum dilution showing inhibition in broth containing $10 \%(\mathrm{v} / \mathrm{v})$ of the stated normal serum; titres for antiserum $\mathrm{I} 869$ are from three separate experiments.

presence of a single dilution of antiserum. Batches of broth with HS, SS or CS were prepared in duplicate, with and without antiserum at a 1/1000 dilution, and warmed to $37^{\circ} \mathrm{C}$ for 10 min. Organisms freshly harvested in the log phase of growth were then added and incubation continued until a $100 \%$ colour change of the medium plus antiserum was observed. During incubation, samples were withdrawn at intervals, and titrated for c.f.u. This experiment was repeated several times and on each occasion growth was linear (Fig. 2). However, the growth rate varied between experiments, and with a standard batch of broth the doubling times varied between 2.5 to $3.5 \mathrm{~h}$. In the absence of antiserum the rate of growth appeared marginally faster for CS and SS than for HS, although the results were not significantly different. Growth in CS broth in the presence of antibody appeared to be similar 


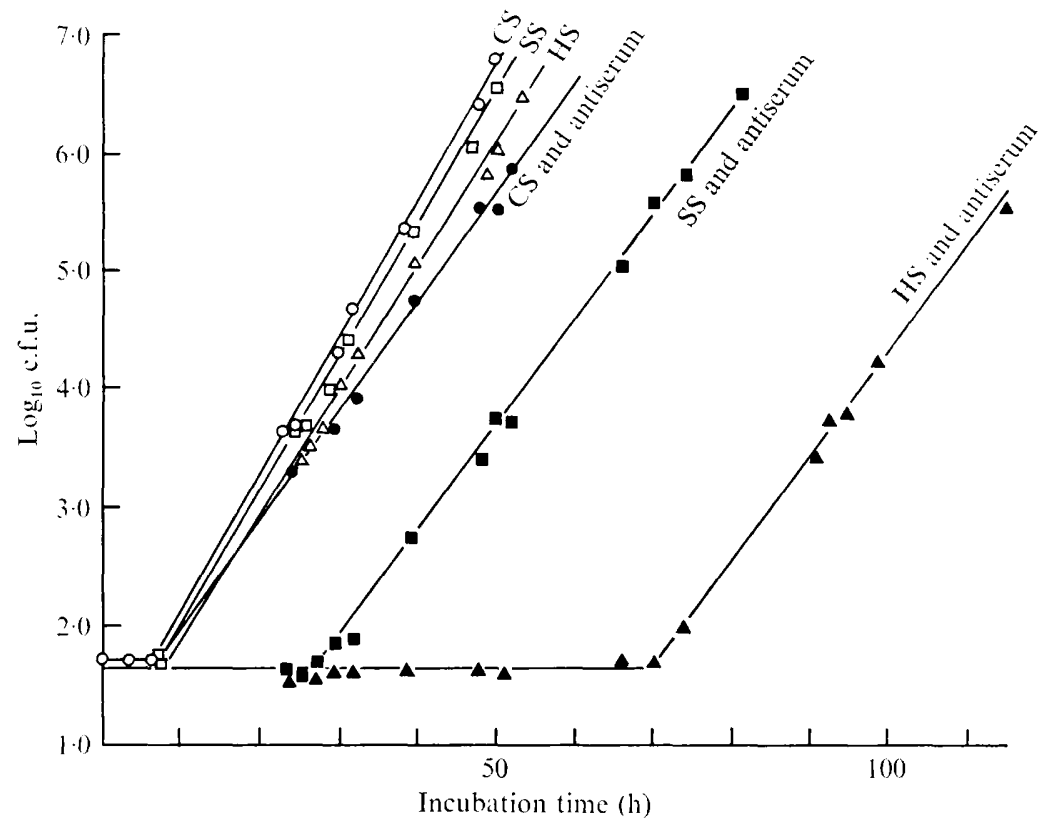

Fig. 2. Growth of Mycoplasma gallisepticum in growth media, containing HS, SS or CS, with and without antiserum. C , CS without antiserum;, CS with antiserum; $\square$, SS without antiserum; $\mathbf{\square}$, SS with antiserum; $\triangle$, HS without antiserum; $\Delta$, HS with antiserum.

to that in CS broth alone, and when CS broth was used for the m.i. test, there was no inhibition with the antiserum at I/1000 dilution, whereas with HS in the medium, metabolic inhibition was observed. Using SS and HS broth the main effect of the antibody was to prolong the lag phase. When an antiserum with a lower early m.i. titre was used, similar growth curves were obtained at a I/100 antiserum dilution but not at I/I000 dilution, again indicating that the effect on growth was a function of antibody concentration. A colour change in the medium was observed when the organism had grown to within $0.5 \log$ of the peak titre.

Antibody absorption from the medium during incubation of organisms in HS broth. The organism appeared to grow normally when the lag phase of growth was over, or when a sample was removed and grown on agar in the absence of antibody. To confirm that inhibition of growth was correlated with the presence of detectable antibody, cultures were prepared with and without antiserum at I/I00 dilution, and the growth curves recorded. At intervals samples were removed from both cultures, the organism inactivated at $56{ }^{\circ} \mathrm{C}$ for $30 \mathrm{~min}$ and the early m.i. titre of the cultures determined. The samples from the control culture showed no m.i. activity, regardless of the stage the culture had reached before inactivation. The early m.i. titre of the culture with antiserum remained constant at $1 / 16$, but became undetectable just before growth commenced (Fig. 3). The result of this experiment was confirmed with another antiserum.

Study of the antigens concerned in the m.i. test. Agglutination of the organism by antibody may have resulted in a quantitative error when determining the c.f.u., whether or not inhibition of growth occurred. However the source of normal serum in the medium influenced this inhibition, suggesting that the antibody may have influenced an essential metabolic activity of the organism. In order to investigate this the antigens responsible for agglutination and those for inhibition of growth were compared. 


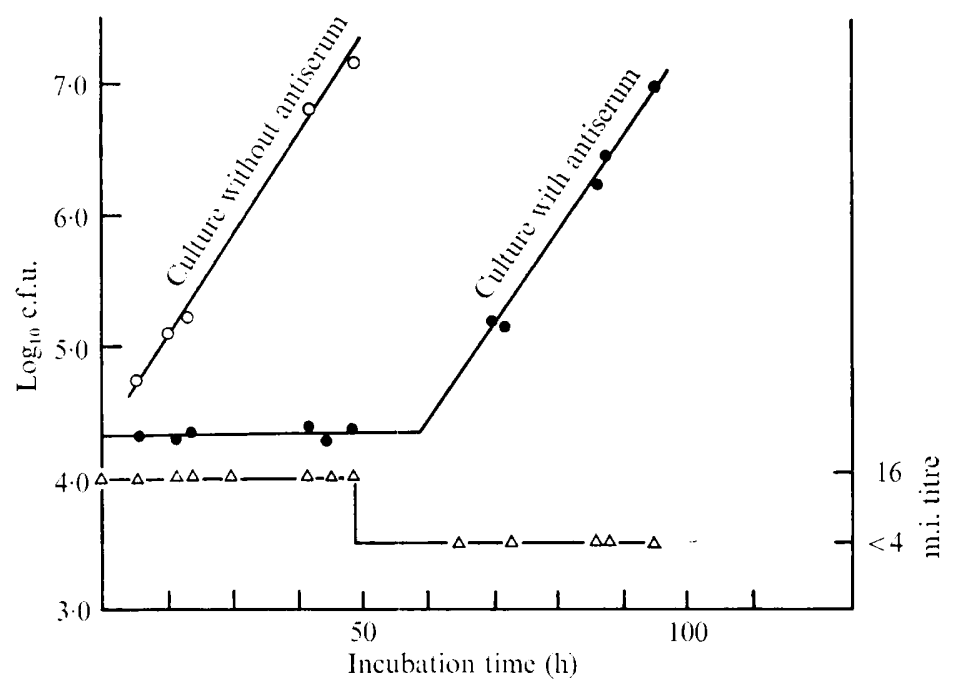

Fig. 3. Growth of Mycoplasma gallisepticum with and without antiserum, correlated with the m.i. titre of the antiserum in the culture sampled during growth. $\bigcirc$, Culture without antiserum; $\bullet$, culture with antiserum; $\triangle$, m.i. titre of the medium containing antiserum.

Table 2. M.i. and agglutination tests with antisera to antigens in medium and organism suspensions

Bird no.

\section{I, 2}

$3,4,5$

$6,7,8$

9, IO, I I

$12,13,14$

$15,16,17$

18,19

20

2 I

22
Source of antigen for antiserum production

Killed organisms from CS chicken broth Killed organisms from HS broth Killed organisms from CS broth Killed organisms from SS broth HS broth alone SS broth alone CS broth alone Live organisms from HS broth Live organisms from SS broth Live organisms from CS broth
Slide agglutination titre* M.i. titre*

40,40
$10,40,40$
$40,40,20$
$20,10,40$
$10,5,20$
$10,20,20$
2,2
40
40
320
$256,5 \mathrm{I} 2$ I28, 1024, 512 $1024,1024,256$ $5 \mathrm{I} 2, \mathrm{I} 28,1024$ all $<2$ all $<2$ $<2,<2$ 2048 I024 2048

* Antisera titrated against organisms grown in the same medium used for preparation of the organism suspensions for immunization; titres expressed as the reciprocal of the highest dilution of antiserum showing agglutination or $\mathrm{m} . \mathrm{i}$.

Organisms were grown in HS, SS, and CS broth and in CS chicken broth. Antisera were prepared in chickens against each medium and against organisms grown in each type of medium, both living and killed. Killed suspensions were prepared as for the agglutination test. The antisera were titrated for m.i. and agglutination, the antigens for each test being prepared in the same medium as that used for antiserum production (Table 2). Antisera to the living and killed organisms showed both m.i. and agglutinating activity. The CS chicken broth alone stimulated no antibody response as measured by m.i. or agglutination, and no precipitating antibody to medium antigens was observed. However, organisms grown in this medium stimulated a good immune response, as tested by m.i. and agglutination. In contrast to these results, antisera to HS, SS and CS broth had agglutination titres but no m.i. activity. 
The organism suspensions grown in CS chicken broth had been prepared by inoculation of the broth with a $10^{-4}$ dilution of the standard organism suspension previously cloned and grown in HS broth. Nevertheless, CS chicken broth organisms, when harvested and concentrated were agglutinated by antiserum to HS broth, even after I I washings in phosphatebuffered saline. However, organisms grown in CS chicken broth from a $10^{-11}$ dilution of the HS broth suspension were no longer agglutinated by antisera to HS broth.

\section{DISCUSSION}

Purcell et al. (1967) demonstrated for several mycoplasmas a close correlation between the dilution of antiserum inhibiting a colour change in the m.i. test, and that inhibiting growth in liquid medium. This finding was confirmed using Mycoplasma gallisepticum by TaylorRobinson \& Berry (I969), and in the present studies where colour change only occurred when organisms had increased substantially in number. Thus growth inhibition and metabolic inhibition may be considered to be synonymous.

The possibility that, following early inhibition of growth by antiserum, the shift from lag phase to growth was due to the appearance of antigenic variants is ruled out by the finding that the new organisms showed a metabolic inhibition by antiserum equal to that of the starting inoculum. The correlation of detectable antibody with maintained lag, and subsequent growth with absence of antibody, together with the capacity of organisms to multiply on transfer to antibody-free medium are consistent with direct interaction of organisms with antibody. It appears probable that limited growth of organisms did occur during the lag phase, and that this was sufficient to absorb the low level of antibody present. Failure to detect this growth could have been due to counting error arising from agglutination of organisms, for at dilutions of antiserum in which m.i. activity persisted, but no direct agglutination was demonstrable, antibodies were detected by the antiglobulin technique using the method of Adler (1967) (unpublished results). The reason that no growth was detected in the presence of high concentration of antibody was not elucidated, but it is probable that in large excess of antibody growth was prevented completely. This conclusion is supported by the work of Barker \& Platt (1967) who showed that the rate of inactivation was proportional to the concentration of antibody in the medium.

The incorporation of medium antigens into the mycoplasma membrane is well recognized (Smith, Dunlop \& Strout, 1966; Jordan \& Kulasegaram, 1968) and Williams \& TaylorRobinson (1967) showed that antigens involved in m.i. were associated with the outer membrane of the organism. In our studies, antiserum to live or killed organisms caused both agglutination and m.i., whereas antiserum to medium antigens caused agglutination only. It is reasonable to conclude that these activities followed association of the antibody with the membrane. This would suggest that there are present on the mycoplasma membrane both antigens from the medium which can agglutinate and also organism-specific antigens, which function both in agglutination and m.i. These latter antigens would appear to be organismspecific because m.i. antibodies were produced in chickens following inoculation of live organisms grown in a homologous chicken medium. Since antisera to medium antigens agglutinate organisms but do not inhibit growth the growth-inhibitory effect of m.i. antibody must be by some mechanism other than agglutination.

Of the various normal sera influencing the inhibitory effect of antiserum it might be supposed that, by the activity of serum factors, such as complement, normal chicken serum would enhance rather than hinder a chicken antigen-antibody reaction. That growth continues normally in the presence of chicken serum, and not horse serum, suggests that m.i. antibody 
reacts with certain membrane 'receptors' concerned with the absorption and utilization of growth nutrients in horse serum, which for some reason are bypassed with chicken serum nutrients. Thus the prolonged lag phase before growth in horse serum, which correlated with the presence of antibody, would result from slow growth whilst these receptors were blocked, until all antibody was utilized.

Contrasting results for the effect of living and killed organisms in the induction of immunity have been reported. Adler, McMartin \& Shifrine (I960) found living organisms to be necessary, whereas Warren, Senterfit \& Siero (I968) produced immunity with large doses of inactivated mycoplasmas. In our experiments, a single dose of living organisms produced antisera of high m.i. potency, whereas several inoculations of inactivated organisms were required for a similar effect. This quantitative difference in dose may reflect the capacity of the viable organisms to provide an effective antigenic stimulus by multiplication within the host.

We thank Professor A. Buxton and Dr John Penhale for their advice, and Mr K. Sommerville for technical assistance.

\section{REFERENCES}

AdLeR, H. E. (1954). A rapid slide agglutination test for the diagnosis of chronic respiratory disease in the field and in laboratory infected chickens and turkeys. Proceedings 9oth Annual Meeting American Veterinary Association, 346.

AdLer, H. E. (1958). A PPLO slide agglutination test for the detection of infectious sinusitis in turkeys. Poultry Science 37, 1116.

ADLER, H. E. (1967). The antiglobulin procedure for the detection of antibodies of avian origin. Avian Diseases II, 69-78.

BARKeR, L. F. \& Platt, J. K. (1967). Role of complement in immune inactivation of Mycoplasma gallisepticum. Journal of Bacteriology 94, 403-408.

Jordan, F. T. C. \& Kulasegaram, P. (I968). Non-specific antibodies in chickens inoculated intratracheally with Mycoplasma gallisepticum. Journal of Comparative Pathology 78, 407-4I4.

MCMartin, D. \& AdLeR, H. E. (I96I). An immunological phenomenon in chickens following infection with Mycoplasma gallisepticum. Journal of Comparative Pathology and Therapeutics 71, 3II-323.

Purcell, R. H., Wong, D., Chanock, R. M., Taylor-Robinson, D., Canchola, A. \& Valdesuso, J. (1967). Significance of antibody to Mycoplasma as measured by metabolic-inhibition techniques. Annals of the New York Academy of Science 143, 664-675.

Smith, S. C., Dunlop, W. R. \& Strout, A. G. (1966). Effect of culture medium on antigenic structure of Mycoplasma. Avian Diseases Io, 173-176.

TAYLOR-RoBInSON, D. \& BERRY, D. M. (I969). The evaluation of the metabolic inhibition technique for the study of Mycoplasma gallisepticum. Journal of General Microbiology 55, 127-137.

Warren, J., Senterfit, L. B. \& Siero, F. (I968). Inactivated culture vaccine against Mycoplasma gallisepticum infection in chickens. American Journal of Veterinary Research 29, 1659-1664.

Williams, M. H. \& TaYlor-Robinson, D. (1967). Antigenicity of Mycoplasma membranes. Nature, London 2I5, 973-974.

Zander, D. V. (1961). Origin of s6 strain of Mycoplasma. Avian Diseases 5, 154-156. 